\title{
Probe into Correlation between Impulsive Aggressive Behavior and Cognitive Impairment in Patients with Bipolar Disorders
}

\author{
Weimin $\mathrm{Wu}^{1}$, Shaohong Zou*, Gaiyu Tong, Yi Zhang \\ ${ }^{1}$ People's Hospital of Xinjiang Uygur Autonomous Region, Uygur 830000, \\ Xinjiang, P.R. China; School of Medicine, Shihezi University, Xinjiang, P.R. \\ China
}

First Author: Weimin Wu, master candidate in School of Medicine of Shihezi University, with research interest in psychological rehabilitation.

*Correspondence: Shaohong Zou. Email: zoushaohong@126.com.

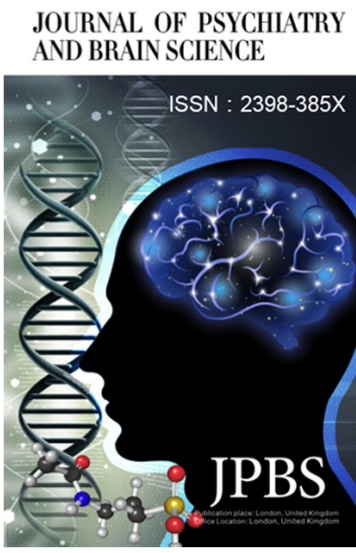

http://jpbs.qingres.com

\section{fOPEN ACCESS}

DOI: 10.20900/jpbs.20160018

Received: July 16, 2016

Accepted: September 22, 2016

Published: October 25, 2016

Copyright: @2016 Cain et al. This is an open access article distributed under the terms of the Creative Commons Attribution License, which permits unrestricted use, distribution, and reproduction in any medium, provided the original author and source are credited.

\begin{abstract}
The studies have found that the impulsive aggressive behaviors may often occur in both incidence stage and remission stage of the patients with disorders, while the social \& psychological factors, co-morbidity and emotional status of the patients with bipolar disorders are closely related with the impulsive aggressive behavior. The studies also indicate a cognitive impairment of different degrees existed in patients with bipolar disorder. The correlation existed between the impulsive aggressive behavior and cognitive impairment in patients with bipolar disorder was systematically described in this paper.
\end{abstract}

Key Words: Bipolar Disorder; Impulsive Aggression; Cognitive Impairment

\section{INTRODUCTION}

Bipolar disorder (BPD) is a kind of multifactor disease with complex clinical manifestation. Characterized by early onset, frequent relapse and chronic outcome, it is correlated with the severe functional impairment, mortality and risk of suicide, including the emotional core abnormity, cognitive deficit, sleeping/waking disorders and highproportion co-morbidity ${ }^{[1]}$. The bipolar disorder was listed by the WHO as the 12th most common mild-severe disabling diseases in the world in 2004 and its lifetime prevalence rate was $4 \%$ in the United States ${ }^{[2]}$.

The bipolar disorder may occur at any age, but it is often started in the patients aged more than 25 years old. The high spirits, increased activities and uninhibited behavior - based manic or hypomanic episodes and the loss of interest and pleasure sense, \& lack of motivation - based depressive episodes are alternated. The average age of symptom incidence: 18 years old for bipolar disorder type I, and 22 years old for bipolar disorder type II. For a long time, the mental health researchers in the world have attached great importance to the 
bipolar disorder due to its relatively high dysfunction, complicated disease course, easy relapse and treatment difficulty.

\section{Research on Impulsive Aggressive Behavior of Patients with Bipolar Disorder}

The impulse is clinically defined as: any tendency that any individual tends to make the rapid and unplanned behavior to himself or others or the behavior for which no adverse impact is considered. According to the definition and etiology of aggressive behavior, the aggressive behavior of the patients with mental illness can be broadly divided into three categories, namely, impulsive aggressive behavior, instrumental aggressive behavior and psychiatric aggressive behavior. The vast majority of aggressive behaviors in patients with bipolar disorders belongs to the impulsive aggressive behavior, mostly occurred during the manic episodes.

Impulse is the common composition and performance in the pathogenesis of bipolar disorders. Several reasons show that the impulse is particularly related to the bipolar disorder type I. First, the impulse is one of the criteria for diagnosis of manic episodes. During the manic episodes, the impulse can increase the risks of destructive behaviors, like uncontrolled consumption, indulgence, and financial investment in danger. Second, the impulse is closely related to the severity of manic symptoms. Finally, the studies ${ }^{[3]}$ show that a high impulsivity can predict the incidence of bipolar disorders and it is associated with the severe disease progression. Therefore, it has been proposed that the impulse represents the core symptom of bipolar disorder, and the impulse remains in different emotional states. Similarly, Stephen M. et al have found that the impulse is not only dependent on the emotional state, but also the constituting features of bipolar disorders ${ }^{[4]}$.

\subsection{Psychosocial Factors of Impulsive Aggressive Behavior in Patients with Bipolar Disorder}

The studies ${ }^{[5]}$ showed that the abuse experiences and parenting style in the childhood of the patients affected the formation of self-esteem and the fear; the family and economic status after growing up decided the quality of the life of the population groups. In addition, long-standing personality characteristics, coping style against the helplessness and self-esteem frustration, negative cognitive style, low social recognition and social support in the environment and other psychosocial features were the reasons leading to the impulsive behavior of bipolar disorder. Similarly, another study ${ }^{[6]}$ showed that less active response and more negative response and decreased social support would be unable to relieve the impact of the outside pressure on the psychology of the patients and they would increase the fear and helplessness to further intensify the impulsive aggression of patients with bipolar disorder.

\subsection{Co-morbidity and Impulsive Aggressive Behavior of Patients with Bipolar Disorder}

A high suicide risk and more body diseases are closely related to the bipolar disorder, including, ischemic heart disease, diabetes, chronic obstructive pulmonary disease (COPD), pneumonia and accidental injuries. Other co-morbid mental disorders were found in about two-thirds of patients with bipolar disorder, such as, anxiety disorders, substance abuse and impulse control disorders. The higher addiction, crime and suicide behaviors, as well as earlier incidence, higher recurrence and more impulse were found in patients with bipolar disorder combined with anti-social personality disorder. The recent studies in this aspect ${ }^{[7]}$ showed a high incidence of aggressive behavior in patients with bipolar disorder. It would further increase the risks of aggressive behavior if the patients with bipolar disorder were accompanied with psychotic symptoms, or a history of suicidal behavior, or co-morbid substance abuse/dependence and personality disorder, or neuropsychological functional defects.

\subsection{Emotion and Impulsive Aggressive Behavior in Patients with Bipolar Disorder}

The studies of Muhtadie, et al ${ }^{[8]}$ suggest that the strong positive emotions might be the important prerequisite for patients with bipolar disorder type I to produce the impulse. The Positive Urgency was the unique impulse variable, and it had no significant correlation with co-morbid anxiety, impulse control, or lifetime substance use disorders. The patients with bipolar disorder were easily influenced by the positive emotions, which may also cause them to have the reckless behaviors. Swann AC, et al. found that, compared to the control group, the preconsciousness and early attention of the patients with bipolar disorder type I produced relatively less stimuli on the brain. The rapid response impulse in patients with bipolar disorder was related to the impaired pre-consciousness inhibition reaction. The impulse was also associated with the poor clinical outcomes of the patients with schizophrenia and bipolar disorder, including substance abuse, suicide behavior and aggression. The bipolar disorder is characterized by impulsive behavior, tendency to increase the rewards and inadequate planning. 
Some evidences suggest that the concrete aspect of impulse has different relationships with two extreme symptoms of emotions, and the impulse of behavior is associated with the manic episodes, while the unplanned impulse is closely associated with the melancholy status ${ }^{[9]}$. Among patients with bipolar disorder, the emotional symptom can exacerbate the impulse. The relevant data show that, compared to the health control group, the patient with bipolar disorder is more impulsive even in the period of stuck period. This means that the impulse can serve as a symptom characteristic of bipolar disorder. Similarly, L. Muhtadie, et al. ${ }^{[10]}$ found that the patients with bipolar disorder type I presented a higher risk of impulsive behaviors while they experienced the strong positive emotions.

Bipolar disorder is a common mental disorder mainly characterized by emotional and behavioral abnormalities. It main incidence status includes depressive episode, manic episode or hypomanic episode. The disease condition of most patients with bipolar disorder can be improved and get controlled after the drug treatment, and their impulsive behavior will be improved and disappear, but the onset is slower.

In summary, the impulsive aggressive behavior will further increase the disease burden of patients with bipolar disorder and it is closely associated with the psychosocial factors, comorbidity and emotional state of the patients. A correct understanding of the impulsive aggressive behavior of patients with bipolar disorder will be of great significance in the diagnosis and treatment of diseases. Weilian Luo, et al. ${ }^{[11]}$ found that the mental nursing intervention made full use of social support systems, which has reduced the induced effect of negative stimulus on the negative emotions in patients. It could quickly and effectively reduce the impulsive behavior in patients, such as, destruction of property, assault, self-injury, suicide, etc., reduce the occurrence of adverse events and reduce the medical risks. With the drug treatment, it will be an effective method to quickly and sustainably control the impulsive behavior of patients with bipolar disorders.

\section{Research on Cognitive Impairment in Patients with Bipolar Disorder}

Cognitive function, also known neurocognitive function, means the ability of brain to process and handle the information, including identification \& integration and processing \& treatment of the information or data, so as to form the comprehensive ability of solving problems and completing the tasks, including, memory, calculation, time \& spatial orientation, structure, language comprehension and expression, enforcement capacity, etc. For over a century, the cognitive impairment in schizophrenia has been recognized. In contrast, the bipolar disorder has only recently been considered as a significant neurocognitive impairment. Burdick KE, et al. ${ }^{[12]}$ expressed that the research of clinical trial design of cognitive impairment in patients with bipolar disorders faced unique challenges. From the concept proof to the determination of clinical efficacy, it is crucial to integrate the challenge of fully solving the unique design modifications with the possible manifestation of cognitive function recovery effect in this trial ${ }^{[12]}$

Some scholars proposed that cognitive impairment is one of main symptoms in patients with bipolar disorder and the possible phenotypic marker of bipolar disorder. In his study using Montreal Cognitive Assessment Scale, Zitong Shen ${ }^{\left[{ }^{[13]}\right.}$ mentioned that the significant attention, memory and execution function and other cognitive impairments existed in patients with bipolar disorder in the stationary phase, and they were related with the duration of disease to certain extent. In the evaluation and contrast using Continuous Performance Test (CPT) and Wisconsin Card Sorting Test (WCST), Zhonggang Wang, et al. ${ }^{[14]}$ also found that the multi-dimensional cognitive impairment existed both in patients with bipolar disorder type I and type II. The impairment degree of patients with bipolar disorder type I in attention disorder, cognitive ability and other aspects was bigger than that of patients with bipolar disorder type II. The impairment of task management ability of patients with bipolar disorder type I was more significant and the attention impairment of patients with bipolar disorder type II was more significant. Through the analysis of literatures of neuropsychological defects of bipolar disorder patients and their first-degree health relatives during the remission period, Bora, et al ${ }^{[15]}$ found that the response inhibition, attention shift, executive function, language memory and sustained attention deficits existed commonly in the patients and their first-degree relatives, while the processing speed, visual memory and verbal expression deficits only existed in the patients with bipolar disorder.

Accordingly, the longitudinal studies showed that many patients experienced the significant subaffective symptoms, and neurocognitive impairment during the acute depression and manic episodes, and the deficits could not be completely relieved even in the stationary phase. In the cognitive domain, the sustained and characteristic impairment was reflected in attention, verbal learning and execution ability, and its average performance decreased by about one criterion compared to the health control group. The studies of Junghee Lee, et al. ${ }^{[16]}$ showed 
a more severe non-social cognitive impairment and a mild social cognitive impairment in patients with bipolar disorders.

The sustained neurocognitive impairment in patients with bipolar disorder will cause serious functional disability, so that the patients are difficult to achieve the full functional recovery. The treatment studies of patients with bipolar disorders mainly focused on the emotional symptoms in the past, but it was also important to consider the substantial disability related to cognitive impairment. Similarly, Shunfa Liu, et al ${ }^{[7]}$ believed that the clinical signs of patients with bipolar disorder were complicated and the clinicians must remember that this disease is characterized by instability and repetition and they could not be fooled by the current representation (manic episode / depression episode) and should attach great importance to the intrinsic evolutional law hidden below the "iceberg". The clinicians should identify, diagnose and treat the patients with potential bipolar disorder as early as possible to help them to pull through and return to society. The bipolar disorder is the episodic course, with normal remission in intermittent episode. If the patients with bipolar disorder can be actively treated, they can be maintained in a stable condition. However, if they cannot be effectively treated for a continuous period, there will be a high recurrence rate. A long-term recurrence will cause a more and more frequent outbreak. The regular intermittent episode will be shortened to lead to a rapid cycling. In such a case, it will be difficult to treat, thus leading to the residual symptoms, chronic condition, personality change and social dysfunction.

Therefore, the cognitive impairment will bring adverse effects on the long-term prognosis and treatment adherence of the patients and it should arouse the attention of the clinicians. With a view to the cognitive impairment in patients with bipolar disorder, the positive treatment will help the recovery of social functions of the patients.

\section{Correlation between Impulsive Aggressive Behavior and Cognitive Functions}

It is now widely recognized that the neurocognitive impairment in patients with bipolar disorder occurs more specifically in the early period of the disease and in the emotional episodes in more and more patients. The cognitive impairment may occur in patients with bipolar disorder type I or bipolar disorder type II. In addition, the meta-data analyses show a poorer cognitive impairment in patients with bipolar disorder with history of mental illness (more common in patient with bipolar disorder type I than that in patient with bipolar disorder type II) than that in patients without history of mental illness, even in emotional remission.

Some scholars proposed to take the neuropsychological defects as the predictive factor of the aggressive behavior in patients with bipolar disorder. Strakowski, et al. ${ }^{[18]}$ found the significant defects of executive function response inhibition, delay satisfaction and attention in patients with bipolar disorder, and a difference of response speed, identification ability and award acquisition in patients with bipolar disorder compared to the control group, which also indicated a defect in executive function, and they found that the behavior of patients was more impulsive.

At present, more and more studies ${ }^{[19,20]}$ show that the cognitive impairment has become one of main symptoms in patients with bipolar disorder and also the important factor affecting social function rehabilitation and disease prognosis of the patients with bipolar disorder. While the aggressive behavior is also one of the common symptoms in patients with bipolar disorder, and it will harm the patient and others, and meanwhile increase psychological and economic burden of the society and the patient family and increase the people's fear and discrimination against the mental illness. A correct understanding of aggressive behavior of patients with bipolar disorders will help to reduce the occurrence of aggressive behavior, while a correct evaluation of the relationship between neurocognitive impairment and impulsive aggressive behavior in patients with bipolar disorder will have a great significance to the patient, its family or the society. It will benefit greatly the individuals and the society to reduce the occurrence of impulsive aggressive behavior by improving the cognition.

In summary, in patients with bipolar disorder, the impulsivity-related neurocognitive process (e.g. attention, inhibition) was also damaged, which further highlights the clinical significance of researching the relationship between them. Currently, there are few clinical studies on whether the increased impulsivity is related to the severity of cognitive impairment, while the evaluation of the relationship between impulsivity and cognitive function in patients with bipolar disorder requires a further discussion.

\section{FUNDING SUPPORT}

This work was supported by grants from the National Natural Science Foundation of China. Program Name: Interactive Study of Impulsive Aggressive Behavior Gene and Psychosocial Factors in Patients with Bipolar Disorder in Uygur and Han Nationality 
in Xinjiang Uygur Autonomous Region (Program Number: 31360239).

\section{REFERENCES}

1. Balanza Martines V, Selva G, Martinez-Aran $A$, et al. Neurocognition in bipolar disorders - a closer look at comorbidities and medications. Eur J Pharmacol. 2010; 626(1):87-96.

2. Price AL, Marana Nissan GR. Bipolar disorders: A review. Am Family Physician, 2012; 85(5):483493.

3. Alloy LB, Abramson LY, Walshaw PD, et al. Behavioral Approach System (BAS) and Behavioral System Inhibition System (BIS) sensitivity and bipolar spectrum disorders: prospective prediction of bipolar mood episodes. Bipolar Disord. 2008; 10:310-322.

4. Stephen M.Strakowski, David E. Fleck, Melissa P.et al. Impulsivity across the course of bipolar disorder. Bipolar Disord. 2010 May; 12(3): 285297. Doi: 10.1111/j.1399-5618.2010.00806.x.

5. Rongrong Luan, Shaohong Zou, Gaiyu Tong, et al. Psychosocial Factor for Impulsive Aggressive Behavior in Patients with Bipolar Disorder. J Int Psy, 1673-2952 (2015), 01-0065-03.

6. Rongrong Luan, Shaohong Zou, Lina Jia, et al. Relationship between Aggressive Behavior and Social Support and Coping Style in Patients with Bipolar Disorder Type II. J Clin Psychol Med, 1005-3220 (2015) 02-0085-04.

7. Wei Cui, Xueyi Wang, Baoping Yan, et al. Aggressive Behavior in Patients with Bipolar Disorder. J Clin Psychol Med, 1005-3200 (2015) 01-0065-02.

8. Muhtadie L, Johnson SL, Carver CS, et al. A Profile Approach to Impulsivity in Bipolar Disorder: the key role of strong emotions. Acta Psychiatr Scand. 2014 February; 129(2):100108. Doi:10.1111/acps.12136.

9. Swann AC, Steinberg JL, Lijffijt $M$, et al. Impulsivity: differential relationship to depression and mania in bipolar disorder. J Affect Disord. 2008; 106:241-248.

10. L.Muhtadie, S.L.Johnson, S.S.Carver, et al. A profile approach to impulsivity in bipolar disorder: the key role of strong emotions. Acta
Psychiatr Scand. 2014 February; 192(2):100108.doi:10.1111/acps.12136.

11. Weilian Luo, Chengcheng Zhang. Clinical Research of Psychological Nursing Intervention on the Impulsive Behavior of Patients with Bipolar Disorder. Today Nurse. 1006-6411 (2014) 11-0126-02.

12. Burdick KE, Ketter TA; Goldberg JF, et al. Assessing cognitive function in bipolar disorder: challenges and recommendations for clinical trial design. The J Clin Psy, 2015 March; 76(3):e342-e350. doi:10.4088/jcp.14cs09399.

13. Zitong Shen. Cognitive Function in Patients with Bipolar Disorder in Stable Condition and Related Factors. China J Health Psychol, 2014; 05-064403.

14. Zhonggang Wang, Shili Ge, Liju Qian, Yuying Cao, Chen Wei, et al. Study on Cognitive Impairment in Patients with Bipolar Disorder and Their First-Degree Relatives. The J Clin Psy, 1005-3220(2014)01-0030-03.

15. Bora E, Yucel M, Pantelis C. Cognitive endophenotypes of bipolar disorder: a metaanalysis of neuropsychological deficits in enthymic patients and their first-degree relatives. J Affect Disord. 2009; 113:1-20.

16. Junghee Lee, Lori Altshuler, Glahn DC, et al. Social and nonsocial cognition in bipolar disorder and schizophrenia: relative levels of impairment. Am J Psychiatry. 2013 March 1; 170(3):.doi:10.1176/appi.ajp.2012.12040490.

17. Shunfa Liu, Qiang Chen, et al. Clinical Research Progress of Bipolar Disorder. Med Recapoitulate, 1006-2084 (2013) 13-2384-03.

18. Strakowski SM, Fleck DE, DelBello MP, et al. Characterizing Impulsivity in Mania. Bipolar Disord. 2009; 11: 41-51.

19. Harvey PD, Wingo AP, Burdick KE, et al. Cognition and Disability in Bipolar Disorder: lessons from schizophrenia research. Bipolar Disord. 2010; 12(4):364 -375.

20. Sole B, Bonnin CM, Torrent C, et al. Neurocognitive impairment across the bipolar spectrum. CNS Neurosci Ther. 2012; 18(3):194200. 